\title{
Construction of Complex Intervention in Traditional Chinese Medicine (TCM): A Preliminary Methodological Study Based on Text Mining and Interviewing Method
}

\author{
Lian Gong, Wenzhi Hao, Feifei Xue* \\ School of Chinese Medicine, Jinan University, Guangzhou, China \\ Email: *Fayexue@126.com
}

How to cite this paper: Gong, L., Hao, W.Z. and Xue, F.F. (2019) Construction of Complex Intervention in Traditional Chinese Medicine (TCM): A Preliminary Methodological Study Based on Text Mining and Interviewing Method. Pharmacology \& Pharmacy, 10, 130-136.

https://doi.org/10.4236/pp.2019.103011

Received: March 4, 2019

Accepted: March 19, 2019

Published: March 22, 2019

Copyright $\odot 2019$ by author(s) and Scientific Research Publishing Inc. This work is licensed under the Creative Commons Attribution International License (CC BY 4.0).

http://creativecommons.org/licenses/by/4.0/

\begin{abstract}
Aim: To explore and analyze the feasibility of establishing a program of complex intervention in Traditional Chinese Medicine (TCM) based on Text Mining and Interviewing method. Methods: According to MRC, Constructing the program of complex intervention in TCM by Text Mining and Interviewing method should include 4 steps: 1) establishment of interview framework via normalization of extraction of ancient documents and Effectiveness of collection of modern periodical literatures; 2) materialization of interview outline based on Focus Group Interview; 3) rudimentary construction of complex intervention program based on Semi-structured Interview; 4) evaluation of curative effect of complex intervention. Conclusions: It is feasible and significative to establish a program of complex intervention in TCM based on Text Mining and Interviewing method.
\end{abstract}

\section{Keywords}

Traditional Chinese Medicine, Text Mining, Interviewing Method, Complex Intervention

\section{Introduction}

The occurrence and development of most diseases are complex processes, which are not only reflected by the different clinical manifestations and transformation of mechanism of pathology of the same disease in different developmental stages, but also because of the close correlation between the disease symptoms and curative effects and multiple dimensions like society, physiology, psychology and 
so on. As a result, complex interventions directed at multiple pathological links, different developmental stages or influence factors of a disease are usually necessary for the clinical practice in order to achieve a satisfactory effect. This kind of complex intervention, of course, can also be defined as an intervention synthesized by multiple intervention measures, including all the intervention measures like drug intervention and non-drug intervention. These measures may be independent from each other, or may be interactive with each other [1].

The inherent concepts of TCM like "unity of heaven and man", "prevention of disease, and "abidance by triple pathogens and syndrome differentiation and treatment" invariably embody its therapeutic ideology of complex intervention and individualized diagnosis and treatment [2]. Thus, based on the TCM view of health and disease and theories of prevention and treatment, from multiple perspectives, to synthesize the merits of various therapeutic methods and to establish a program of complex intervention are suitable for the developmental characteristics of disease, which are also necessary means to improve the clinical effect.

\section{Methods}

According to the framework published by MRC (Medical Research Council) [3] in 2000 , the complex intervention program may be established and evaluated by four steps: pre-clinical theoretical preparation and exploration, model research (based on literature and qualitative research, selection of primary components and preliminary construction of complex intervention model, including the confirmation of research elements and mutual relationship), trial test (pilot experiment on the rudimental model), confirmatory test (design and confirm the RCT program of complex intervention program) and observation of popularization and application (long-term implementation and evaluation). In the light of TCM features, this paper is both qualitative and quantitative and intended to discuss the feasibility of establishing the TCM complex intervention by the method of textual analysis and semi-structured interview, of which the specific steps are as follows(as shown in Figure 1):

\begin{tabular}{|l|l|}
\hline $\begin{array}{l}\text { Interview Framework } \\
\text { ancient documents } \\
\text { modern periodical literatures } \\
\text { investigation standard :normalization } \\
\text { and effectiveness }\end{array}$ & $\longrightarrow \begin{array}{l}\text { Establishment Interview } \\
\text { Outline } \\
\text { back-to-back questionnaire } \\
\text { interviewer screening } \\
\text { interview content selection }\end{array}$ \\
\hline $\begin{array}{l}\text { Efficacy Evaluation } \\
\text { the classical randomized controlled } \\
\text { trial } \\
\text { the pragmatic randomized controlled } \\
\text { trial }\end{array}$ & $\begin{array}{l}\text { Semi-structured Interview } \\
\text { Implementation } \\
\text { interview scope } \\
\text { interviewees and content } \\
\text { individualized patient interview }\end{array}$ \\
\hline
\end{tabular}

Figure 1. To construct the basis of TCM comprehensive intervention research. 


\subsection{Step 1: Establishment of Interview Framework}

Literature learning is the basis for every kind of TCM research. The interview framework of complex intervention can be also rudimentarily established by massive text mining and extraction of effective information. Text mining is developed from the data mining, but it doesn't mean that, text mining can be realized by a simple application of data mining technology to the text collection [4]. Instead, it needs an expertise of medical profession for text retrieval and intelligent analysis. The content of literature learning includes ancient documents and modern literatures, attention should be paid to the following issues in the process of establishing interview framework.

\subsubsection{Normalization of Extraction of Ancient Documents}

There are a tremendous amount of TCM ancient documents, which contain the experiences of diagnosis and treatment of innumerable excellent medical practitioners. In a post-genome age, numerous scholars are still obsessed in the study on ancient documents, which is sufficient evidence that the document research is very important for the modernized study on TCM. However, Chinese culture is long-standing and well-established, due to the richness of implication of Chinese language and polysemy, synonym and other issues, one TCM symptom may be described by different words and some terms are unclear and undefined, which is adverse to the extraction and conclusion of information. Moreover, given the update of technology of diagnosis and treatment and change in disease spectrum, many potentially effective methods are gradually ignored and even forgotten. In an age of big data, the application of multidisciplinary technology to mine the ancient documents and maximize their values is an indispensable step for modernization of TCM [5]. The researchers have already introduced the quantitative method, shifting from the single-frequency analysis to multi-factor complex analysis and correlation analysis, transiting from the simple statistical analysis to the application of data mining technology, such as using the grounded theory and content analysis to extract the etiology, pathogenesis, symptom and treatment related to diseases [2]; using the multivariate statistics methods like factor analysis to summarize the therapeutic principles of disease [6]; using the method of multi-label learning and random forest to choose the symptoms and vital signs and differentiate TCM syndromes [7].

\subsubsection{Effectiveness of Collection of Modern Periodical Literatures}

The modern periodical literatures are also the crystallization of experiences of all medical practitioners. With the formulation of various standards, the literatures about theoretical discussion of TCM are increasingly written in a normalized manner. However, due to the particularity of TCM itself, some clinical research literatures are written in a poor quality. To ensure the accuracy of complex intervention program, and also to conduct an effective evaluation of clinical effect in late stage, according to the requirement of evidence-based medicine that, the research quality of methodology and evidence management should be treasured, 
the literatures should undergo a quality control when they are included, such as RoB tools, Jadad scale or modified Jadad scale, and so on, only the high-quality research can be included for information extraction. The interventional systematic evaluation of a certain TCM treating method can also be done by the use of Meta-analysis and other means. The high-quality literatures are more representative, and able to extract the TCM concepts, syndromes, diagnostic criteria and intervention measure for a certain disease more accurately, providing a theoretical basis for the implementation of interview and formulation of complex intervention program.

\subsection{Step 2: Materialization of Interview Outline Based on Focus Group Interview}

The establishment of interview outline can be usually done through Delphi method, which is to consult the predictive opinions of members of panel of experts by a means of back-to-back questionnaire. But the content of questionnaire is fully settled, which cannot reflect the syndrome differentiation thinking of clinician completely. Thus, the focus group interview method, which acquires the first-hand information from experts in a face-to-face manner, is recommended here. The specific method is to select some representative participants for a progressive and guided interview specific to a certain problem, and the interview usually lasts for 2 - 3 hours [8].

Based on the content of this study, the selected interviewee should include clinicians, experts of syndrome science, social methodology researchers, statisticians, members of research group and so on, the interview framework should be revised to finally complete a semi-open interview outline composed of several dimensions like informed consent, general information, disease-related contents, treatment-related contents, suggestions and advices.

\subsection{Step 3: Rudimentary Construction of Complex Intervention Program Based on Semi-Structured Interview}

The method of semi-structured [9] interview is an important method for social scientific qualitative research, which requires that, the interview subject or content should be unsettled and the scope of interview should be decided by outline or sketchy questions, which tends to obtain massive information within a short time. Based on the focus group interview, the construction of TCM complex program is prepared from the perspective of patient's need by one-to-one individualized patient interview. The relatives who are very familiar with the patient's condition may also be taken as an interviewee. This process can be alternated with the interview of focus group, the interview outline should be modified according to the key points of patient interview, finally achieving information saturation and preliminarily forming a theoretical hierarchical structure of TCM complex intervention that is multi-dimensional and comprehensive. 


\subsection{Step 4: Evaluation of Curative Effect of Complex Intervention}

Complex intervention is an objective circumstance that runs through the entire clinical medical activity of TCM, and appears in the process of treatment, prevention, rehabilitation and nursing of disease [10]. The development or outcome of patient's condition is affected to a great extent by the active ingredients of these complex drug interventions and non-drug interventions and interrelationship between them.

Randomized Controlled Trials (RCT) has already been accepted universally as the gold standard for research method of clinical test. Among which, the classical Randomized Controlled Trial (RCT), namely, Explanatory Randomized Controlled Trials has already been widely used for the clinical research of new TCM drugs. But the traditional explanatory RCT is confronted by a challenge and limitation brought by methodology when it is used to evaluate the comprehensive therapeutic program of TCM [11].

Some international experts of RCT methodology proposed another test method, namely, the Pragmatic Randomized Controlled Trial, the former develops the connotation of traditional explanatory RCT. It takes the relationship between "People" and evaluation into account to a greater extent, and stresses the patient-based clinical outcome report. The intervention settings take the effect of doctor on outcome evaluation into account and the doctor is permitted to be inclined therapeutically. According to the consideration of constraint brought by medical ethics in the control settings, the placebo control may not be used. This people-oriented design feature increases the compliance for long-term therapy and is more consistent with the study on TCM complex intervention theoretically [12].

In this study, we selected 8 patients from various regions to conduct interviews, as showed in Table 1. We developed a diagnostic and treatment procedure based on the outline and results of the semi-structured interview method, and applied it to the patient's treatment. A total of 60 patients were dealt with and identified as the intervention group. Similarly, the current basic clinical treatment was applied to patients with the same disease, a total of 60 patients were processed, that is, the control group. The results of the satisfaction survey of two of them are as follows in Table 1 and Table 2.

Table 1. Basic information on interviewed patients in semi-structured interviews.

\begin{tabular}{cccc}
\hline Interviewee & Gender & Region & Treatment direction \\
\hline Interviewee 1 & Male & Guangzhou & Medical disease \\
Interviewee 2 & Male & Guangzhou & Complex diseases \\
Interviewee 3 & Female & Beijing & PMS \\
Interviewee 4 & Male & Guangzhou & Medical diseases \\
Interviewee 5 & Female & Beijing & PMS \\
Interviewee 6 & Female & Fujian & PMS \\
Interviewee 7 & Male & Beijing & Medical diseases \\
Interviewee 8 & Male & Henan & Medical diseases \\
\hline
\end{tabular}


Table 2. Comparison of satisfaction with the diagnosis and treatment process and results of the control group and the intervention group.

\begin{tabular}{cccccccccc}
\hline Group & No. & \multicolumn{2}{c}{ Satisfied } & \multicolumn{3}{c}{ Basic satisfaction } & Dissatisfied & Satisfaction \\
\cline { 3 - 7 } & of cases & $\mathrm{n}$ (example) & $\%$ & $\mathrm{n}$ (example) & $\%$ & $\mathrm{n}$ (example) & $\%$ & $(\%)$ \\
\hline $\begin{array}{c}\text { Intervention } \\
\text { group }\end{array}$ & 60 & 44 & 73.33 & 16 & 26.67 & 0 & 0.00 & 100.0 \\
\begin{tabular}{c} 
Control group \\
\hline
\end{tabular} & 60 & 26 & 43.33 & 24 & 40.00 & 10 & 16.67 & 83.33 \\
\hline
\end{tabular}

\section{Conclusions}

G. L. Engel pointed out in 1977 that, in order to understand the determinant of disease, and achieve a reasonable mode of therapy and health care, medical model should consider the patient, the environment where patient lived and supplementary system that was designed by society to deal with the destructive effect of disease, namely, the function of doctor and health care system. That is to say, the most effective treatment for any kind of disease is a multi-dimensional complex intervention.

TCM is a treasure of Chinese nation [13]. In an age of biology-psychology-social medical model like today [14], the ideologies of treatment based on syndrome differentiation like "people oriented", "abidance by triple pathogens" and "correspondence between man and universe" clinically show their inherent features of complex intervention. However, both the selection of research method and design of research program have been misunderstood to a certain degree in terms of either research on normalization of syndromes or formulation of intervention measure and evaluation of curative effects, namely, equating the objectification with the quantification, leading to a great difficulty in exerting the field of advantage of TCM completely. It is more conducive to establish a TCM-characterized system of diagnosis and treatment if the qualitative research methods like interview methods can be introduced to the study on modernization of TCM and combined with the quantitative methods like textual analysis and data mining on the basis of complex critical thinking patterns such as combination of disease differentiation and syndrome differentiation of TCM, concurrent consideration of symptomatic relief and radical treatment, laying equal stresses on part and whole and regulation of the body and mind.

\section{Acknowledgements}

This project was supported by grants from the National Natural Sciences Foundation of China (No. 81503496) and fund for Scientific Research cultivation and innovation of Jinan University (No. 21617464).

\section{Conflicts of Interest}

The authors declare no conflicts of interest regarding the publication of this paper. 


\section{References}

[1] Dowding, D.W., Cheyne, H.L., Hundley, V., et al. (2011) Complex Interventions in Midwifery Care: Reflections on the Design and Evaluation of an Algorithm for the Diagnosis of Labor. Midwifery, 27, 654-659. https://doi.org/10.1016/j.midw.2009.11.001

[2] Lao, Y.R. (2008) Preliminary Study on the Methodology of Construction of TCM Complex Intervention Program for Rheumatoid Arthritis. Doctoral Dissertation, Guangzhou University of TCM, Guangzhou.

[3] Medical Research Council (2000) A Framework for Development and Evaluation of RCT's for Complex Interventions to Improve Health. MRC, British, 3.

[4] Zheng, S.M., Hu, L.S., Li, Y.L., Cheng, S.S. and Wang, W. (2007) Study on the Theory of Holism of Body and Spirit by the Use of Text Mining. Chinese Archives of Traditional Chinese Medicine, 25, 2480-2481

[5] Xue, F.F., Wang, L.M. and Chen, J.X. (2013) Exploration and Analysis on the Philological Research Thought of TCM Syndrome. Journal of TCM, 54, 918-920.

[6] Xue, F.F. and Chen, J.X. (2010) Study on Ancient Literatures Based on a Factor Analysis on Syndrome Characteristics of Liver Depression. Chinese Archives of Traditional Chinese Medicine, 28, 988-990.

[7] Xu, W.P., Gu, W.J. and Liu, G.P. (2016) Study on the Selection of Solid Syndrome Features and Classification and Identification of Syndromes of Chronic Gastritis Based on Method of Random Forest and Multi-Label Learning Algorithm. Chinese Journal of Information on Traditional Chinese Medicine, 23, 18-23.

[8] Liu, J.P. (2006) Methodology of Clinical Research of Evidence-Based TCM. 2nd Edition, People's Medical Publishing House, Beijing.

[9] Liu, Y., Zhang, Z. and Zhang, H.Y. (2011) Study on the Construction of Normalized Theoretical Framework of TCM Syndrome Differentiation by the Use of SemiStructured Interview Method. Chinese Archives of Traditional Chinese Medicine, 29, 738-743.

[10] Xie, Y.M., Zhi, Y.J. and Wang, Y.Y. (2008) First Exploration of New Method Suitable for Evaluation of Clinical Effect of TCM-Method of Evaluating the Clinical Effect of Complex Intervention Measure. Journal of TCM, 49, 395-397.

[11] Fei, Y.T., Yang, H. and Liu, J.P. (2008) Pragmatic Randomized Controlled Trial and Its Application in the Field of TCM. Journal of TCM, 49, 116-119.

[12] Roland, M. and Turgeon, D.J. (1998) Understanding Controlled Trials: What Are Pragmatic Trials? BMJ, 316, 285. https://doi.org/10.1136/bmj.316.7127.285

[13] Leung, B. (2015) Traditional Chinese Medicine: The Human Dimension. Routledge, Abingdon-on-Thames. https://doi.org/10.4324/9781315656373

[14] Zhao, J.-W. and Feng, Q. (2015) Analysis on Current Status and Plights of Translational Medicine. Journal of International Translational Medicine, 3, 59-66. 